\title{
Julian Schwinger and the Semiclassical Atom
}

\author{
Berthold-Georg Englert \\ Centre for Quantum Technologies \\ and Department of Physics, National University of Singapore; \\ MajuLab, Singapore; \\ cqtebg@nus.edu.sg
}

\begin{abstract}
In the early 1980s, Schwinger made seminal contributions to the semiclassical theory of atoms. There had, of course, been earlier attempts at improving upon the ThomasFermi model of the 1920s. Yet, a consistent derivation of the leading and next-to-leading corrections to the formula for the total binding energy of neutral atoms,

$$
-\frac{E}{e^{2} / a_{0}}=0.768745 Z^{7 / 3}-\frac{1}{2} Z^{2}+0.269900 Z^{5 / 3}+\cdots,
$$

had not been accomplished before Schwinger got interested in the matter; here, $Z$ is the atomic number and $e^{2} / a_{0}$ is the Rydberg unit of energy. The corresponding improvements upon the Thomas-Fermi density were next on his agenda with, perhaps, less satisfactory results. Schwinger's work not only triggered extensive investigations by mathematicians, who eventually convinced themselves that Schwinger got it right, but also laid the ground, in passing, for later refinements - some of them very recent.
\end{abstract}

\section{Introduction}

Julian Schwinger's groundbreaking work on quantum electrodynamics first and then, more generally, on quantum field theory and the physics of elementary particles is, of course, at the center of his legacy. In addition, he made seminal contributions to many other topics, among them the semiclassical theory of atoms. This work of his is summarized in an essay of 1985 for non-expert readers that did not get published in his lifetime. ${ }^{1}$ I shall recall here some aspects of our collaboration from mid 1981 to early 1985 and also mention later work that was triggered by Schwinger but did not involve him.

\section{Schwinger's papers of 1980 and 1981}

While teaching a course on quantum mechanics in the late 1970s, Schwinger was intrigued by the Thomas-Fermi (TF) model and the systematic deviation of the TF approximation for the total binding energy of an atom from the Hartree-Fock (HF) values (curve a and circles in Fig. 1). This was crying out for an understanding. Schwinger responded to the challenge with his derivation (in $1980^{2}$ ) of the leading correction that had been conjectured by John Scott in 1952, $\frac{3}{3}$ now known as the Scott correction. Schwinger did not know Scott's paper before a referee pointed it out.

Although the inclusion of the Scott correction improved the binding energies substantially (curve b in Fig. 1), there remained a systematic error. In a paper of 


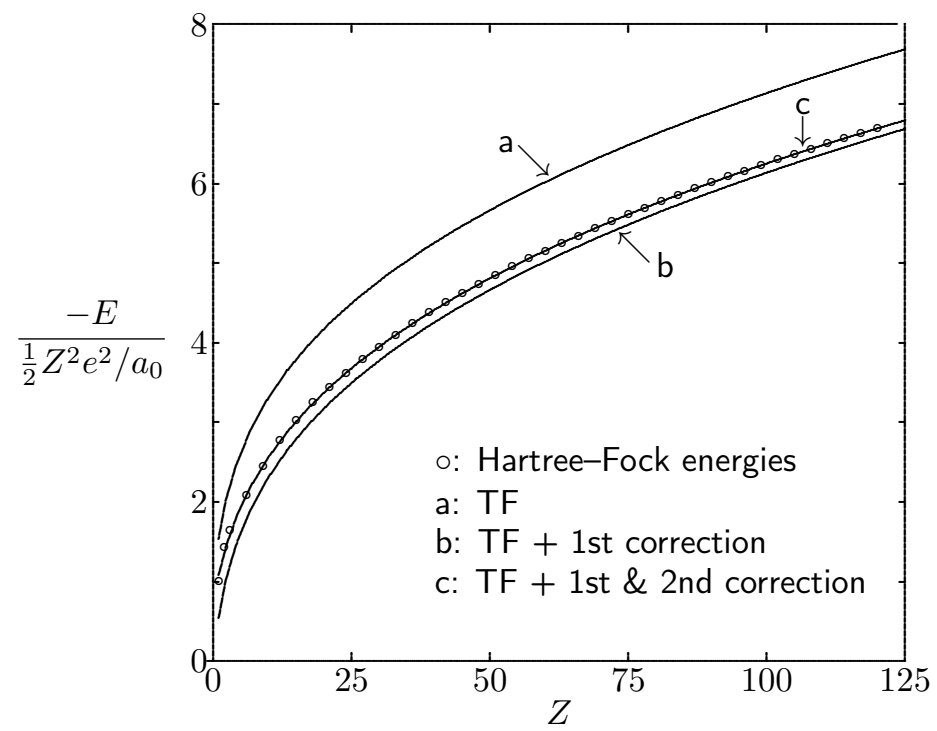

Fig. 1. Total binding energy of neutral atoms, as a function of the atomic number $Z$, in Rydberg units $\left(e^{2} / a_{0}\right)$ and scaled by $\frac{1}{2} Z^{2}$. The circles represent the Hartree-Fock values, and curves a, $\mathrm{b}$, and c show, respectively, the Thomas-Fermi approximation by itself, and with the leading and the next-to-leading corrections included. See also Fig. 8 in Ref. 1.

1981, 4 Schwinger took care of this as well, again giving a clear-cut demonstration of what had been conjectured before; 5 this takes us to curve $\mathrm{c}$ in Fig. 1 that goes right through the circles of the HF values. The consecutive approximations are the three terms in Eq. (46) of Ref. 1, which are recalled in the Abstract.

\section{Mathematicians in action}

Schwinger's derivation of the two leading corrections to the TF energy of atoms in Refs. 2 and 4 convinces any theoretical physicist, but the standards of mathematical physics are different. Earlier, in 1977, Elliot Lieb and Barry Simon had given a proof that the TF approximation is asymptotically exact as $Z \rightarrow \infty$; see Ref. $[$ for the precise statement. Now, two other duos of mathematicians dealt with Scott's leading correction and Schwinger's next-to-leading correction.

First, Heinz Siedentop and Rudi Weikard in a series of papers ${ }^{7} 11$ published 1986-1989 showed that Scott's correction is indeed the leading correction when $Z$ is large enough. Then, Charles Fefferman and Luis Seco devoted another series of papers $\frac{12}{20}$ to the second correction, published 1989-1995, eventually confirming that Schwinger's result is correct.

These three important pieces of mathematical research illustrate how, in the history of this subject matter, the link between theoretical physics and mathematics has been a one-way road: The physicists provide conjectures and the mathematicians convert them into theorems. There hasn't been any benefit for the physicists 
in return, beyond being assured that they got it right (which, by their own standards, they knew already). The work by Lieb and Simon did not yield a suggestion on how to improve on the TF approximation, nor did the Siedentop-Weikard proof indicate how to go beyond the Scott correction, and an analogous remark applies to the accomplishments by Fefferman and Seco. While it is true that the task chosen by each of the three duos was limited to proving what was conjectured, it would have been also nice to get an idea about where to look for the next improvement.

\section{Summer of 1981: A homework assignment}

Schwinger finished the work on the corrections to the TF energy by the end of 1980 (Ref. 4 was submitted in December 1980), and then began studying the corresponding modifications of the TF density. Lester DeRaad had already provided numerical solutions to a modification of the TF differential equation,, 421

$$
\frac{\mathrm{d}^{2} \phi}{\mathrm{d} y^{2}}=\frac{\phi^{3 / 2}}{y^{1 / 2}}+\phi
$$

with

$$
\phi(0)=\frac{48 \pi}{(22)^{3 / 2}} Z=1.461 Z \text { and } \phi(\infty)=0,
$$

where $\phi(y)$ is the auxiliary function in terms of which the quantities of interest can be computed; see Ref. 21 for details. As expected, this improved on the TF approximation for the electron density at large distances - the values obtained for diamagnetic susceptibilities, essentially the squared distance from the nucleus weighted by the density, were much better than the TF values - but, so the paper summarizes, the "outcome of this test [...] hardly warrants proclaiming a successful conclusion to the search for an extrapolation of the TF model into the outer regions of the atom."

Accordingly, this search continued and led Schwinger to considering

$$
\frac{\mathrm{d}^{2} \phi}{\mathrm{d} y^{2}}=y\left(\sqrt{\frac{\phi}{y}}+\frac{1}{3}\right)^{3}
$$

with

$$
\phi(0)=1.461 Z \quad \text { and } \quad \phi\left(y_{0}\right)=0, \quad 1.461(Z-N)=-y_{0} \frac{\mathrm{d} \phi\left(y_{0}\right)}{\mathrm{d} y_{0}},
$$

which he gave to me as a homework assignment in the summer of 1981 (see Fig. 2). At this time, he spent part of a sabbatical leave in Tübingen, hosted by my Ph.D. supervisor Walter Dittrich. I had recently submitted my thesis and was waiting for the defense, and so I was available when Schwinger needed someone with programming skills. It was easy to get the numbers, such as a table of $y_{0}$ values for different $Z$ and $N$, and the numbers passed the various tests that Schwinger could subject them to. 


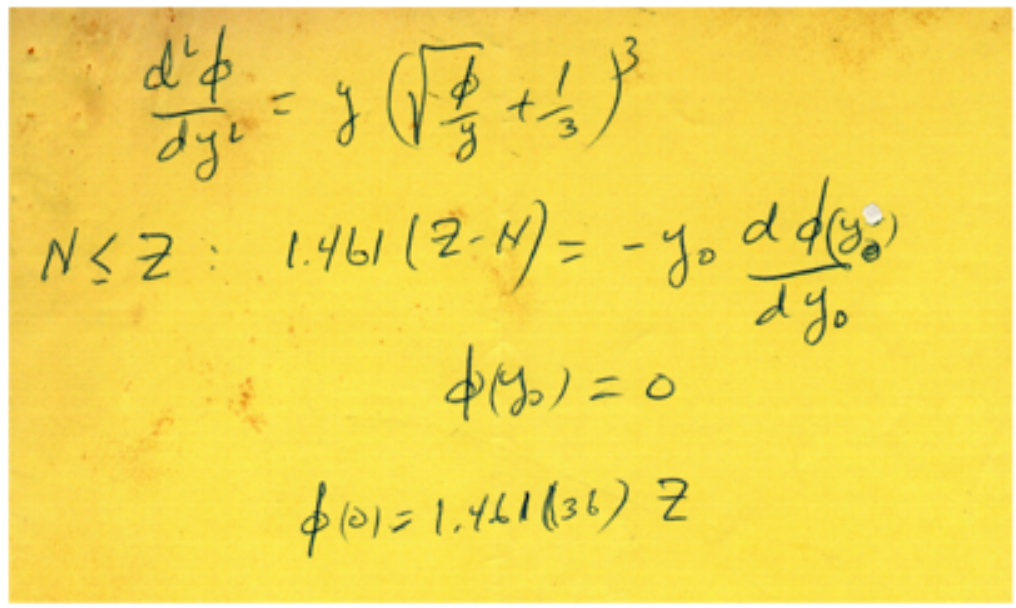

Fig. 2. The homework assignment: Schwinger's modification of the TF differential equation in his hand writing. The white spot is a drop of correction fluid.

He then gave me a copy of the draft paper he was working on, and I finally understood the origin of Eq. (3) and the physical meaning of $\phi(y)$. While my feedback on the draft and other input did have a bearing on the published version, 22 it was hardly enough to earn co-authorship. Schwinger, however, thought differently and my objection to being first author were gently and firmly pushed aside: "There is much to be said in favor of the alphabet."

The electron densities from the formalism based on Eq. (3) do improve on the TF approximation. They give reasonably good values for the diamagnetic susceptibilities, better than the HF numbers for larger atoms ${ }^{*}$ and this was good motivation for taking the next step.

\section{1984 and 1985: Two trios of papers}

By the time Ref. 22 was finalized and submitted, I had been recruited by Schwinger as a postdoc, and we were already working on the follow-up project that yielded a trio of papers in 1984.23 .25

Equations (1) and (3) were modifications of the TF equation that incorporated Paul Dirac's approximate exchange energy ${ }^{26}$ and Carl Friedrich von Weizsäcker's inhomogeneity correction, 27 both responsible for the second correction, but not Scott's leading correction. While this is acceptable when the focus is on the outer reaches of the atom, it is somewhat inconsistent.

Scott's correction addresses the failure of the TF approximation in the vicinity of the nucleus where the singularity of the Coulomb potential results in large changes of the potential energy over short distances. Therefore, it was necessary to treat

*In those days, HF numbers were systematically too large by about $10 \%$. 
the strongly bound electrons differently.23] While Dirac's exchange energy could be used without any essential modification, Weizsäcker's inhomogeneity correction required a refinement that accounts for higher-order terms, and Schwinger designed an ingenious method for that, where a certain averaging ${ }^{\dagger}$ over simpler Weizsäckertype expressions is performed. ${ }^{24}$ An extensive numerical study confirmed that the resulting modification of the TF model was worth the effort. ${ }^{25}$

It is, however, not truly satisfactory because of an unresolved problem with the handling of the strongly bound electrons. That involves a separation on the energy scale, and the electrons with a binding energy less than the threshold value are treated in the TF fashion (with modifications). The electrons with larger binding energy are regarded as dominated by the Coulomb potential of the nucleus, with corrections accounted for by perturbation theory. As a consequence of the rather different treatments, there is an unphysical dependence on the choice of the threshold value. While this can be systematically removed in the resulting correction to the energy (this is an important detail in Schwinger's derivation of Scott's correction ${ }^{2}$ ), we did not manage to get rid of it in the density f $^{7}$

Meanwhile, we were wondering about the difference between the HF energies in Fig. 1 and those of curve c. As Figs. 9 and 10 in Ref. 1 show, this difference is a notso-regular oscillatory contribution, which suggests that the degeneracy associated with energy shells - Niels Bohr's shells ${ }^{31}$ modified by the repulsive forces between electrons - is important here. This suggestion was not misleading and eventually provided an understanding of these energy oscillations, reported in another trio of papers, published in 1985.32 . 34 .

Here, a crucial observation is that the TF approximation can be obtained by first expressing the energy as a sum over WKB energies, and then replacing the sum by an integral. ${ }^{32}$ This integral is a zeroth-order approximation of the sum, and the hierarchy of higher-order terms contains oscillatory terms that can be extracted systematically. The leading oscillatory term contributes ${ }^{34}$

$$
\begin{aligned}
& -0.4805 Z^{4 / 3} \frac{e^{2}}{a_{0}} \sum_{k=1}^{\infty} \frac{(-1)^{k}}{(\pi k)^{3}} \sin \left(2 \pi k \lambda_{0}\right) \\
= & 0.3206\left\lceil\lambda_{0}\right\rfloor\left(\frac{1}{4}-\left\lceil\lambda_{0}\right\rfloor^{2}\right) Z^{4 / 3} \frac{e^{2}}{a_{0}}
\end{aligned}
$$

to the energy, where $\lambda_{0}=0.928 Z^{1 / 3}$ and $\left\lceil\lambda_{0}\right\rfloor$ denotes the difference between $\lambda_{0}$ and its nearest integer; see also Eq. (63) in Ref. 1. After the TF energy $\left(\propto Z^{7 / 3}\right)$, Scott's leading correction $\left(\propto Z^{6 / 3}\right)$, and Schwinger's second correction $\left(\propto Z^{5 / 3}\right)$, this contribution $\left(\propto Z^{4 / 3}\right)$ is the next in line. As of today (2019), there is no

\footnotetext{
${ }^{\dagger}$ Strictly speaking, it is not an average because the terms are weighted by the Airy function $\operatorname{Ai}(x)$, which is assuredly positive for $x>0$ but not for $x<0$. See Ref. 28 for a recent benchmarking exercise.

${ }^{\ddagger}$ The situation is remarkably different in momentum space where the Scott-corrected density does not suffer from this problem. $29[30$
} 


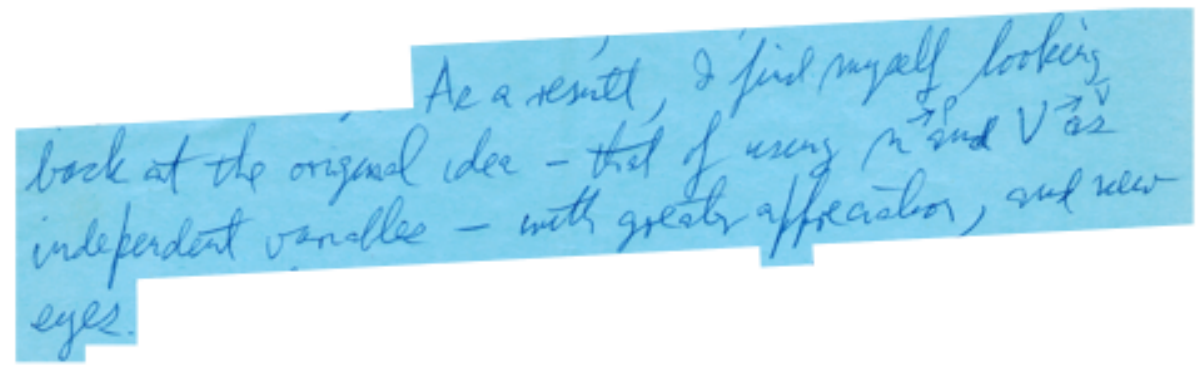

Fig. 3. Schwinger's appreciation, in 1981, of functionals with both the density and the potential as independent variables.

mathematical proof of its correctness, and so we have to be content with the evidence presented in Ref. 34 .

Consult Ref. 35 for a detailed account of all these developments.

\section{Functionals of both the density and the effective potential}

The argument presented in Ref. 22 proceeds from a functional for the energy that has the single-particle density, the electrostatic potential, and the chemical potential as independent variables. Schwinger wrote this down without giving a derivation but justifying it by its consequences, and I must have just accepted it. It is indeed consistent with a remark by Schwinger in a letter of 1981 that he wrote in response to the detailed feedback I gave on the draft paper mentioned above; see Fig. 3. I am pretty sure that I did not appreciate the flexibility of this approach at the time. In hindsight, I regard the functional in Ref. 22 as very clever and just right for the purpose at hand, but not as a useful starting point for more general developments.

We did not use anything quite like this again and, instead, switched to a formalism based on energy functionals of the particle density, the effective potential, and the chemical potential as independent variables. The effective potential is the sum of the external potential (here: the Coulomb potential of the nucleus) and an interaction potential obtained from the response of the interaction energy (here: direct and exchange electrostatic energy) to variations of the density. This was a natural thing to do, it was intuitive, and no justification seemed necessary - it was clearly an obvious matter for Schwinger, an example of his phenomenal intuition about physics and its mathematical language.

As for myself, I took some time to comprehend fully what was so obvious to him. Eventually, at the time of writing Ref. [36, with a precursor in Ref. 35, I saw how this is systematically connected to the standard density-functional theory as formalized by Pierre Hohenberg and Walter Kohn ${ }^{37}$ — and this turned the densitypotential functional into a central tool for all of my subsequent work on related topics. The connection is actually quite simple: just subject the kinetic energy term in the Hohenberg-Kohn density functional to a Legendre transformation. 
The concept of density-potential functionals is a contribution of lasting value, perhaps Schwinger's most important contribution to the field. These functionals are more flexible than density-only functionals, and they facilitate systematic approximations that go beyond formal gradient expansions, for which the Weizsäcker term of $1935^{27}$ is the prototype. In particular, there is a density-potential functional for the Scott-corrected TF model but no density-only functional.

As another example, I mention the somewhat amusing case of gradient corrections to the TF model in two dimensions. Papers in the early 1990s established or so it seems - that there are no such corrections, all terms in a formal power series of the density gradient have vanishing coefficients; see, for example, Refs. 38 and 39. On the other hand, the TF approximation to the kinetic energy is not exact, whether in one, two, or three dimensions, and so one has the puzzling situation of a non-exact approximation with all systematic correction terms vanishing. The puzzle disappears as soon as one recognizes that there are nonzero gradient corrections in the density-potential functional, and they can be evaluated perturbatively. $\underline{40}$

There is also an analog of the density-potential functionals in momentum space, where one gets an energy functional with the momentum-space density, the effective kinetic energy, and the chemical potential as independent variables. The hierarchy is repeated: We have the momentum-space version of the TF model ${ }^{36}$ (which has succumbed to the power of mathematics ${ }^{41}$ ), of the Scott-corrected TF model,, 30 and of a model with exchange energy and gradient corrections included as well. $\underline{42}$

Finally, there is a recent, novel attempt at approximating the potential part of the density-potential functional. There is a connection with the single-particle propagator that was already exploited in Ref. 24 and led then to the Airy-averaged expressions mentioned above, with an improvement over a related method by Eugene Wigner $\underline{43}$ and John Kirkwood. $\stackrel{44}{ }$ Now, one can alternatively approximate the propagator by a factorization into terms that refer only to the kinetic energy or only to the potential energy - a technique introduced and developed by Hale Freeman

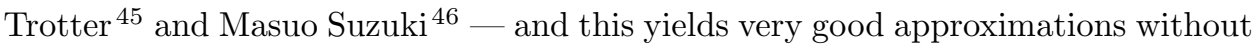
a gradient expansion. $\frac{47}{47}$ The applications thereof are work in progress.

\section{Summary}

Schwinger's papers of 1980 and 1981 on the leading and the second correction to the TF energy are well known and have triggered extensive mathematical studies. His insight that one should treat the density and the effective potential as independent variables on equal footing is just as important and crucial for ongoing research.

\section{Acknowledgments}

Julian Schwinger, always kind and generous, taught and guided me from 1981 till his premature death in 1994. His lessons, both formal and informal, were invaluable. I was a greenhorn when he recruited me as his postdoc at UCLA. When I moved to the University of Munich forty months later, I was a physicist. 


\section{References}

1. J. Schwinger and B.-G. Englert, The Statistical Atom, eprint arXiv:1807.10109 [quant$\mathrm{ph}](2018)$.

2. J. Schwinger, Thomas-Fermi model: The leading correction, Phys. Rev. A 22, 1827 (1980).

3. J. M. C. Scott, The Binding Energy of the Thomas-Fermi Atom, Philos. Mag. 43, 859 (1952).

4. J. Schwinger, Thomas-Fermi model: The second correction, Phys. Rev. A 24, 2353 (1981).

5. G. I. Plindov and I. K. Dmitrieva, Notes on the nonrelativistic binding energy for neutral atoms, Phys. Lett. 64A, 348 (1978).

6. E. H. Lieb and B. Simon, The Thomas-Fermi theory of atoms, molecules and solids, Adv. Math. 2322 (1977).

7. H. Siedentop and R. Weikard, On the leading energy correction for the statistical model of the atom: Non-interacting case, Abh. Braunschweig Wiss. Ges. 38, 145 (1986).

8. H. Siedentop and R. Weikard, Upper bound on the ground state energy of atoms that proves Scott's conjecture, Phys. Lett. 120A, 341 (1987).

9. H. Siedentop and R. Weikard, On the leading energy correction for the statistical model of the atom: interacting case, Comm. Math. Phys. 112, 471 (1987).

10. H. Siedentop and R. Weikard, On the leading correction of the statistical atom: lower bound, Europhys. Lett. 6, 189 (1988).

11. H. Siedentop and R. Weikard, On the leading correction of the Thomas-Fermi model: lower bound, Invent. Math. 97, 159 (1989).

12. C. L. Fefferman and L. A. Seco, An upper bound for the number of electrons in a large ion, Proc. Natl. Acad. Sci. USA 86, 3464 (1989).

13. C. L. Fefferman and L. A. Seco, The Ground-State Energy of a Large Atom, Bull. AMS 23, 525 (1990).

14. C. L. Fefferman and L. A. Seco, Asymptotic Neutrality of Large Ions, Comm. Math. Phys. 128, 109 (1990).

15. C. L. Fefferman and L. A. Seco, Eigenvalues and Eigenfunctions of Ordinary Differential Operators, Adv. Math. 95, 145 (1992).

16. C. L. Fefferman and L. A. Seco, Aperiodicity of the Hamiltonian Flow in the ThomasFermi Potential, Revista Math. Iberoamericano 9, 409 (1993).

17. C. L. Fefferman and L. A. Seco, On the Dirac and Schwinger Corrections to the Ground-State Energy, Adv. Math. 107, 1 (1994).

18. C. L. Fefferman and L. A. Seco, The Eigenvalue Sum for a One-Dimensinal Potential, Adv. Math. 108, 263 (1994).

19. C. L. Fefferman and L. A. Seco, The Density in a One-Dimensional Potential, Adv. Math. 107, 187 (1994).

20. C. L. Fefferman and L. A. Seco, The Density in a Three-Dimensional Radial Potential, Adv. Math. 111, 88 (1995).

21. L. L. DeRaad, Jr., and J. Schwinger, New Thomas-Fermi theory: A test, Phys. Rev. A 25, 2399 (1982).

22. B.-G. Englert and J. Schwinger, Thomas-Fermi revisited: The outer regions of the atom, Phys. Rev. A 26, 2322 (1982).

23. B.-G. Englert and J. Schwinger, Statistical atom: Handling the strongly bound electrons, Phys. Rev. A 29, 2331 (1984).

24. B.-G. Englert and J. Schwinger, Statistical atom: Some quantum improvements, Phys. Rev. A 29, 2339 (1984). 
25. B.-G. Englert and J. Schwinger, New statistical atom: A numerical study, Phys. Rev. A 29, 2353 (1984).

26. P. A. M. Dirac, Note on exchange phenomena in the Thomas atom, Proc. Cambridge Philos. Soc. 26, 376 (1930).

27. C. F. von Weizsäcker, Zur Theorie der Kernmassen, Z. Phys. 96, 431 (1935).

28. M.-I. Trappe, Y. L. Len, H. K. Ng, and B.-G. Englert, Airy-averaged gradient corrections for two-dimensional Fermi gases, Ann. Phys. (NY) 385, 136 (2017).

29. K. Buchwald and B.-G. Englert, Thomas-Fermi-Scott model: Momentum-space density, Phys. Rev. A 40, 2738 (1989).

30. M. Cinal and B.-G. Englert, Thomas-Fermi-Scott model in momentum space, Phys. Rev. A 45, 135 (1992).

31. N. Bohr, On the Constitution of Atoms and Molecules, Philos. Mag. 26, 1 (1913).

32. B.-G. Englert and J. Schwinger, Semiclassical atom, Phys. Rev. A 32, 26 (1985).

33. B.-G. Englert and J. Schwinger, Linear degeneracy in the semiclassical atom, Phys. Rev. A 32, 36 (1985).

34. B.-G. Englert and J. Schwinger, Atomic-binding-energy oscillations, Phys. Rev. A 32, 47 (1985).

35. B.-G. Englert, Semiclassical Theory of Atoms, Lecture Notes in Physics $\mathbf{3 0 0}$ (Springer, 1988).

36. B.-G. Englert, Energy functionals and the Thomas-Fermi model in momentum space, Phys. Rev. A 45, 127 (1992).

37. P. Hohenberg and W. Kohn, Inhomogeneous electron gas, Phys. Rev. 136, B864 (1964).

38. A. Holas, P. M. Kozlowski, and N. H. March, Kinetic energy density and Pauli potential: dimensionality dependence, gradient expansions and non-locality, J. Phys. A: Math. Gen. 24, 4249 (1991).

39. J. Shao, Exact kinetic energy functional of noninteracting fermions, Mod. Phys. Lett. B 7, 1193 (1993).

40. M.-I. Trappe, Y. L. Len, H. K. Ng, C. A. Müller, and B.-G. Englert, Leading gradient correction to the kinetic energy for two-dimensional fermion gases, Phys. Rev. A 93, 042510 (2016).

41. H. Siedentop and V. von Conta, Statistical Theory of the Atom in Momentum Space, Markov Processes and Related Fields 21, 433 (2015).

42. M. Cinal and B.-G. Englert, Energy functionals in momentum space: Exchange energy, quantum corrections, and the Kohn-Sham scheme, Phys. Rev. A 48, 1893 (1993).

43. E. Wigner, On the Quantum Correction For Thermodynamic Equilibrium, Phys. Rev. 40, 749 (1932).

44. J. G. Kirkwood, Quantum Statistics of Almost Classical Assemblies, Phys. Rev. 44, 31 (1933).

45. H. F. Trotter, On the product of semi-groups of operators, Proc. Amer. Math. Soc. 10, 545 (1959).

46. M. Suzuki, Generalized Trotter's formula and systematic approximants of exponential operators and inner derivations with applications to many-body problems, Commun. Math. Phys. 51, 183 (1976).

47. T. T. Chau, J. H. Hue, M.-I. Trappe, and B.-G. Englert, Systematic corrections to the Thomas-Fermi approximation without a gradient expansion, New J. Phys. 20, 073003 (2018). 